\title{
Funkzellenabfrage muss eingeschränkt werden!
}

\section{Konferenz der Datenschutzbeauftragten des Bundes und der Länder, Entschließung vom 27. Juli 2011}

Presserklärung des Bayerischen Landesbeauftragten für den Datenschutz, Dr. Thomas Petri, als Vorsitzender der Konferenz der Datenschutzbeauftragten des Bundes und der Länder:

\section{Voraussetzungen für Funkzellen- abfragen strenger fassen!}

Die Konferenz der Datenschutzbeauftragten des Bundes und der Länder fordert den Bundesgesetzgeber auf, Konsequenzen aus der Dresdner Funkzellenabfrage zu ziehen.

Im Zuge der sogenannten „Dresdner Funkzellenabfrage" hatten sächsische Sicherheitsbehörden knapp eine Million Datensätze von Handybesitzern ausgewertet, die am 18. und 19. Februar 2011 in der Innenstadt Dresdens ihr Handy benutzt hatten.

In der Entschließung vom 27.07.2011 weisen die Datenschutzbeauftragten des Bundes und der Länder darauf hin, dass die in Dresden erfolgte Funkzellenabfrage leider keinen Einzelfall darstellt, sondern auf strukturellen Mängeln der Befugnisnorm in der Strafprozessordnung beruht. Die einschlägige Vorschrift des $₫ 100 \mathrm{~g}$ Strafprozessordnung ist nämlich so allgemein gefasst, dass sie die massenhafte Erfassung von Menschen gestattet, die keinen Anlass für einen staatlichen Eingriff gegeben haben.

Daher fordert die Konferenz der Datenschutzbeauftragten des Bundes und der Länder den Bundesgesetzgeber auf, die Voraussetzungen des $\$ 100 \mathrm{~g}$ Strafprozessordnung klarer und vor allem strenger zu fassen.

\section{Entschließung: Funkzellenabfrage muss eingeschränkt werden!}

Die Strafverfolgungsbehörden in Dresden haben mit einer sog. Funkzellenabfra- ge anlässlich von Versammlungen und dagegen gerichteter Demonstrationen am 19. Februar 2011 Hunderttausende von Verkehrsdaten von Mobilfunkverbindungen erhoben, darunter die Rufnummern von Anrufern und Angerufenen, die Uhrzeit sowie Angaben zur Funkzelle, in der eine Mobilfunkaktivität stattfand. Dadurch sind zehntausende Versammlungsteilnehmerinnen und Versammlungsteilnehmer, darunter Abgeordnete von Landtagen und des Deutschen Bundestages, Rechtsanwältinnen und Rechtsanwälte, sowie Journalistinnen und Journalisten in Ausübung ihrer Tätigkeit, aber auch Anwohnerinnen und Anwohner der dicht besiedelten Dresdener Innenstadt, in ihrer Bewegung und ihrem Kommunikationsverhalten erfasst worden. Dieser Vorfall verdeutlicht die Schwäche der gesetzlichen Regelung. Rechtsgrundlage der nichtindividualisierten Funkzellenabfrage ist bisher $\$ 100 \mathrm{~g}$ Abs. 2 S. 2 StPO, wonach im Falle einer Straftat von erheblicher Bedeutung eine räumlich und zeitlich hinreichend bestimmte Bezeichnung der Telekommunikation ausreichend sein soll, um Verkehrsdaten bei den Telekommunikationsdiensteanbietern erheben zu dürfen. Diese Aussage wird mit einer allgemeinen Subsidiaritätsklausel verknüpft. Diese 2001 in die Strafprozessordnung eingefügte Regelung ist unzureichend, da sie weder hinreichend bestimmt ist noch den heutigen technischen Gegebenheiten entspricht. Aktuelle Geräte erzeugen durch ihren Datenverkehr ohne aktives Zutun des Besitzers eine Vielzahl von Verkehrsdaten, die später in einer Funkzellenabfrage erhoben werden können.

Die Funkzellenabfrage ist ein verdeckter Eingriff in das Fernmeldegeheimnis (Art. 10 GG). Sie richtet sich unterschiedslos gegen alle in einer Funkzelle anwesenden Mobilfunkgerätebesitzer, nicht nur - wie etwa eine Telekommunikationsüberwachung nach $\$ 100$ a StPO - gegen bestimmte einzelne Tatverdächtige. Sie offenbart Art und Umstände der Kommunikation von $\mathrm{u}$. U. Zehntausenden von Menschen, die selbst keinen Anlass für einen staatlichen Eingriff gegeben haben. Sie schafft damit des Weiteren die Möglichkeit, diese Personen rechtswidrig wegen Nicht-Anlasstaten, etwa Verstößen gegen das Versammlungsgesetz, zu verfolgen. Sie ist bezogen auf einzelne Personen ein Instrument der Verdachtsgenerierung. Die Strafprozessordnung regelt nicht näher, wie die Behörden mit den erhobenen Daten umzugehen haben, insbesondere nicht, über welche Zeiträume, zu welchen Personen und in welchen anderen Zusammenhängen die erhobenen Daten polizeilich weiter verwendet werden dürfen.

Das Bundesverfassungsgericht hat stets betont, dass die Erhebung von Verkehrsdaten erhebliche Rückschlüsse auf das Kommunikationsverhalten zulässt. Verkehrsdaten können das soziale Netz des Betroffenen widerspiegeln; allein aus ihnen kann die Verbindung zu Parteien, Gewerkschaften oder Bürgerinitiativen deutlich werden.

Die Konferenz der Datenschutzbeauftragten des Bundes und der Länder fordert daher den Bundesgesetzgeber auf, den Anwendungsbereich für eine nichtindividualisierte Funkzellenabfrage einzuschränken, dem Grundsatz der Verhältnismäßigkeit zu stärkerer Beachtung in der Praxis zu verhelfen, das Erforderlichkeitsprinzip zu stärken (etwa durch die Pflicht zur unverzüglichen Reduzierung der erhobenen Daten auf das zur Strafverfolgung oder gerichtlichen Auseinandersetzung Erforderliche) sowie die Löschungsvorschrift des $\S 101$ Abs. 8 StPO zu präzisieren. 